Please do not remove this page

RMIT

UNIVERSITY

\title{
Sensitivity analysis of process variations for resonantly enhanced modulators on LiNbO3
}

Nguyen, Giang; Mitchell, Arnan

https://researchrepository.rmit.edu.au/esploro/outputs/9921862297601341/filesAndLinks?institution=61RMIT_INST\&index=null

Nguyen, G., \& Mitchell, A. (2006). Sensitivity analysis of process variations for resonantly enhanced modulators on LiNbO3. IEEE Journal of Lightwave Technology, 24(5), 2199-2206.

https://doi.org/10.1109/JLT.2006.872270

Published Version: https://doi.org/10.1109/JLT.2006.872270

Repository homepage: https://researchrepository.rmit.edu.au

(c) 2006 IEEE. Personal use of this material is permitted. However, permission to reprint/republish this material for advertising or promotional purposes or for creating new collective works for resale or redistribution to servers or lists, or to reuse any copyrighted component of this work in other works must be obtained from the IEEE.

Downloaded On 2023/04/26 18:48:07 +1000 


\title{
Sensitivity Analysis of Process Variations for Resonantly Enhanced Modulators on $\mathrm{LiNbO}_{3}$
}

\author{
Thach Giang Nguyen, Yuvaraja S. Visagathilagar, Member, IEEE, and Arnan Mitchell, Member, IEEE
}

\begin{abstract}
A sensitivity analysis on the performance of resonantly enhanced modulators on $\mathrm{X}$-cut $\mathrm{LiNbO}_{3}$ using a thick coplanar-waveguide electrode structure due to the fabrication tolerances is presented. The distributions of the various parameters to the performance fluctuation are analyzed. It is shown that a large-gap electrode makes the modulators less sensitive to the variations of electrode dimensions.
\end{abstract}

Index Terms-Optical communication, optical modulation, resonance, sensitivity.

\section{INTRODUCTION}

$\mathbf{M}$ ACH-ZEHNDER optical modulators (MZM) on $\mathrm{LiNbO}_{3}$ have been widely used in optical-communication systems. Modulators with a very large bandwidth have been demonstrated. These modulators usually employ a traveling-wave electrode structure. In many telecommunication applications including radio-on-fiber [1], photonic oscillator [2], photonic mixing [3], optical time-division multiplexing [4], and advanced modulation format, only a narrow bandwidth is required. Thus, for these applications, the modulation efficiency over a narrow bandwidth can be enhanced using resonant electrode structures.

Recently, several resonant-type modulators have been proposed and demonstrated [5]-[7]. These modulators provide significant improvements in modulation efficiency over conventional traveling-electrode modulators. In [8], a numerical optimization technique was applied to the design of the electrode structure used in resonantly enhanced modulators on $\mathrm{X}$-cut $\mathrm{LiNbO}_{3}$. For a resonant electrode structure, the degree of the electric-field enhancement is mainly determined by the attenuation of the resonant cavity. At the same time, the inherent switching voltage of the modulator should be reduced to achieve the best possible efficiency.

The investigation in [8] suggests that optimal electrode configurations are those with thick electrodes. Thick electrodes reduce the electrode loss and the microwave propagation constant. Therefore, the degree of the resonant enhancement is increased with thick electrodes. The electrodes are usually formed by electroplating of gold on the substrate with the patterns defined by a photoresist layer. With the current elec-

Manuscript received October 4, 2005; revised February 7, 2006

The authors are with the Microelectronics and Materials Technology Centre, School of Electrical and Computer Engineering, Royal Melbourne Institute of Technology, Melbourne, Vic. 3001, Australia (e-mail: s9510300@ student.rmit.edu.au; yuvaraja@rmit.edu.au; arnan.mitchell@rmit.edu.au).

Digital Object Identifier 10.1109/JLT.2006.872270 troplating technology, gold electrodes with thicknesses of up to $30 \mu \mathrm{m}$ can be realized. However, the $30-\mu \mathrm{m}$ plating process may have somewhat large process variation. This causes a fluctuation in the characteristics of the fabricated modulator electrodes. Because of the resonant nature of the electrode, the operating frequency and the modulation efficiency of a modulator that utilizes a resonant electrode strongly depend on the characteristics of the electrode. Furthermore, due to the high-Q resonant cavity, the modulation efficiency reduces very quickly when the operating frequency moves away from the electrode resonant frequency. Therefore, to ensure high fabrication yield, it is important that the resonant frequency is relatively insensitive to fabrication variability.

In this paper, we investigate the effects of the real-world manufacturing tolerances on the performance of the resonantly enhanced modulators, including the resonant frequency and the modulation efficiency at the operating frequency. The characteristics of the fabricated modulator electrodes are calculated using FEM models, and then, the resonant performance is evaluated. In [8], it was found that although a large electrode gap makes the inherent switching voltage higher, the resonant enhancement that can be achieved with a large-gap modulator is higher than that of a narrow-gap modulator. Therefore, resonant modulators with a large electrode gap provide higher modulation efficiency at the resonant frequency than those with a smaller gap with approximately the same electrode lengths. Here, it will be shown that modulators with large electrodes are also most tolerant of the fabrication fluctuations.

\section{Resonantly Enhanced Modulator AND OPTICAL RESPONSE}

By placing the terminations on both input and output ports of the modulator electrode to form a Fabry-Pérot-type cavity [9], most of the microwave signal undergoes multiple reflections inside the cavity to form resonant standing waves. This process results in an enhanced electric field inside the modulator electrode. Following [8], the terminations are simply modeled as inductors when calculating the modulator response.

The electrode of the modulator is an electroplated-gold coplanar-waveguide (CPW) structure, as shown in Fig. 1. Ti-diffused optical waveguides are formed in an X-cut and Y-propagation $\mathrm{LiNbO}_{3}$. To minimize the coupling, the optical waveguides are separated by $26 \mu \mathrm{m}$. With that opticalwaveguide separation, the center electrode has $22-\mu \mathrm{m}$ width to achieve the lowest possible inherent switching voltage. The buffer-layer thickness and electrode thickness are 0.2 and $30 \mu \mathrm{m}$, respectively. 


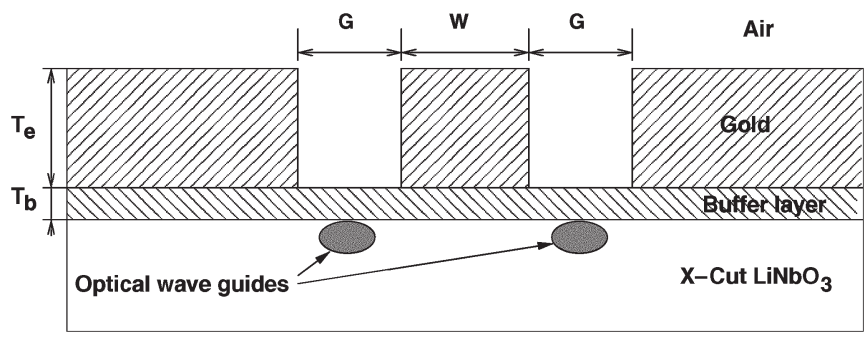

Fig. 1. Modulator cross section.

With the input and output terminations attached to form the resonant cavity, the optical response of a resonantly enhanced modulator is given by Visagathilagar et al. [10]

$$
\mathrm{OR}(f)=\frac{\pi}{V_{\pi} L} \int_{0}^{L} V(z, f) e^{j\left(\frac{2 \pi f}{c}\right) n_{0} z} d z
$$

where $V_{\pi}$ is the dc switching voltage of the modulator without the terminations, $L$ is the total length of the active section, $V(z, f)$ is the complex voltage at point $z$ along the active section of the electrode at frequency $f, n_{0}$ is the effective index of the guided optical mode, and $c$ is the speed of light in free space.

When considering the push-pull configuration of a symmetrical modulator, the dc switching voltage can be calculated as

$$
V_{\pi} L=\frac{\lambda_{0} G}{n_{e}^{3} r_{33} 2|\Gamma|}
$$

where $\lambda_{0}$ is the optical wavelength in free space, $G$ is the gap between electrodes, $n_{e}$ is the extraordinary refractive index of the substrate, $r_{33}$ is the pertinent electrooptic coefficient of $\mathrm{LiNbO}_{3}$, and $\Gamma$ is the overlap integral factor between the microwave electric field and the optical power distribution.

The optical response of a resonantly enhanced modulator has a peak at the microwave resonant frequency and quickly rolls off at other frequencies. The rate of the roll off depends on the Q-factor of the resonant cavity. For a given operating frequency $f_{0}$ and electrode configuration, the terminations and electrode length $L$ can be optimized to achieve microwave resonance at the frequency $f_{0}$. With fixed terminations and cavity length, the resonant frequency is largely determined by the microwave effective index of the electrode. Therefore, to keep the modulator operating at the designed frequency $f_{0}$, the variation of the microwave effective index needs to be kept as small as possible. Any variation of the electrode cross-sectional dimensions will cause the microwave effective index, together with the characteristic impedance, attenuation constant, and the inherent switching voltage to change. This in turn causes the shift of the resonant frequency and the deviation of the modulator optical response.

\section{NUMERICAL EXPERIMENTS}

In the following sections, an analysis of the sensitivity of the modulator characteristics to the variations of the fabrication parameters as could be expected in a real-world manufacturing process will be presented. Two optimized modulator electrode configurations will be considered: one with a very large electrode gap $(G=50 \mu \mathrm{m})$ and one with moderate gap $(G=20 \mu \mathrm{m})$. The designed resonant frequency is $10 \mathrm{GHz}$. The characteristics of the modulator electrode, including the microwave effective index, characteristic impedance, attenuation constant, and the inherent switching voltage for different variations of the electrode cross-sectional dimensions from their nominal values, are calculated. Then, the performance of the resulting resonantly enhanced modulators is predicted using a simulation model [10].

In order to accurately model the characteristics of thick CPW electrodes, a full wave FEM simulator [11], [12] is used. The microwave effective index, characteristic impedance, attenuation constant, and the electrooptic overlap factor $\Gamma$ are then calculated from the FEM simulation.

\section{A. Variations of the Electrode Gap and Width}

In this section, the effects of the electrode gap and width variations on the performance of resonantly enhanced modulators will be investigated. The sizes of the electrode gap and width can change from the designed value due to the tolerance of the mask manufacture, photoresist development, and electroplating processes.

Fig. 2(a) and (b) shows the derivation of the microwave effective index from the nominal value when the fabricated electrode gap and width vary by $\pm 4 \mu \mathrm{m}$ from their designed values $G-W-G=20-22-20$ and $G-W-G=50-22-50 \mu \mathrm{m}$, respectively. The buffer-layer thickness and the electrode thickness are fixed at their designed values. The comparison of Fig. 2(a) and (b) reveals that in the case of the designed electrode gap of $20 \mu \mathrm{m}$, the effective index is more sensitive to the electrode gap and width variations than the case of $50-\mu \mathrm{m}$ designed electrode gap.

In Fig. 3(a) and (b), the contours of the variation of the peak resonant frequency, as a function of the electrode gap and width variation, are plotted. Fig. 3(a) and (b) closely resembles Fig. 2(a) and (b). In the case of the electrode with moderate gap $(G=20 \mu \mathrm{m})$, the peak resonant frequency can vary up to $3 \%$ from the designed frequency when the gap and width are varied by $\pm 4 \mu \mathrm{m}$, whereas with large electrode gap $(G=50 \mu \mathrm{m})$, the maximum deviation of the peak resonant frequency is about $2 \%$. It can also be seen that the peak resonant frequency does not change if the electrode gap and width vary in complimentary ways, e.g., the peak resonant frequency is maintained when the width increases by $2 \mu \mathrm{m}$ and the gap decreases by $2.5 \mu \mathrm{m}$ for moderate-gap configuration. This will be the case if the width and gap variations result from overetching or underetching of the photoresist-layer formation used in the electroplating process.

When the electrode gap and width change from their designed values, the electrode characteristic-impedance, attenuation, and inherent switching voltage will also change from their nominal values. These changes will cause the variations of the modulation efficiency. Fig. 4 presents the deviation of the modulator optical response from the designed value at the operating frequency. For a large gap, the impact of the variation of electrode gap and width on inherent switching voltage, 


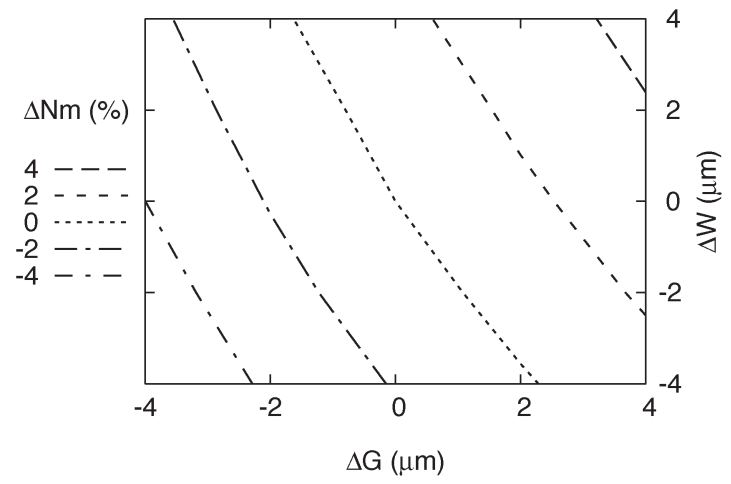

(a)

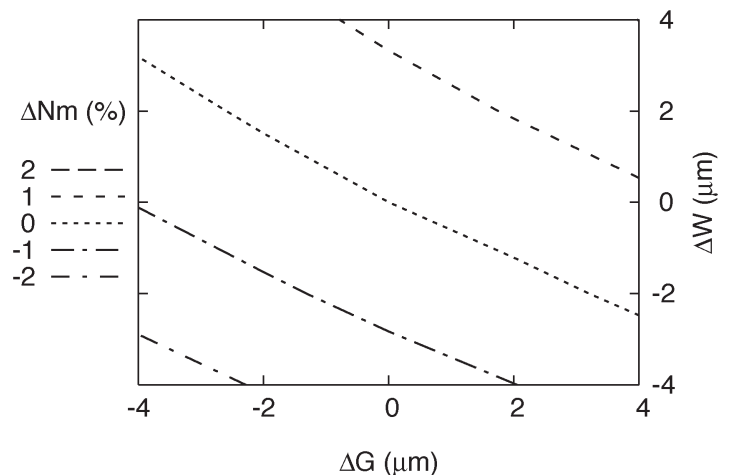

(b)

Fig. 2. Effects of $G-W$ variations on the electrode-microwave effective index $\mathrm{Nm} . \Delta \mathrm{Nm}=\left(\mathrm{Nm}-\mathrm{Nm}_{0}\right) / \mathrm{Nm}_{0} * 100$, where $\mathrm{Nm}_{0}$ is the effective index when the gap and width are at their nominal values. (a) Nominal $G=20 \mu \mathrm{m}$ and (b) nominal $G=50 \mu \mathrm{m}$.

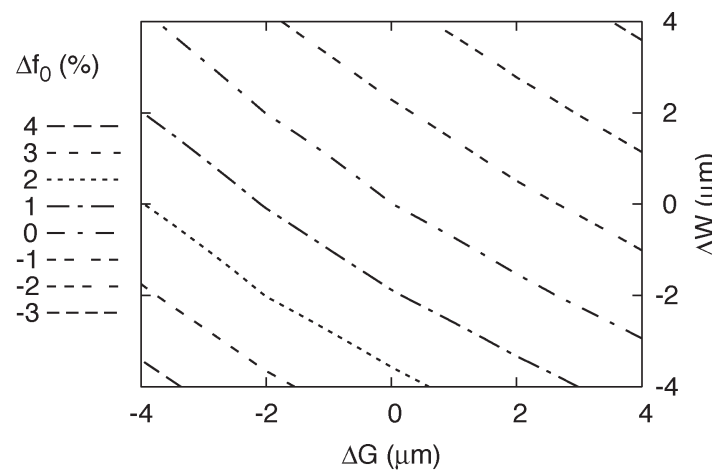

(a)

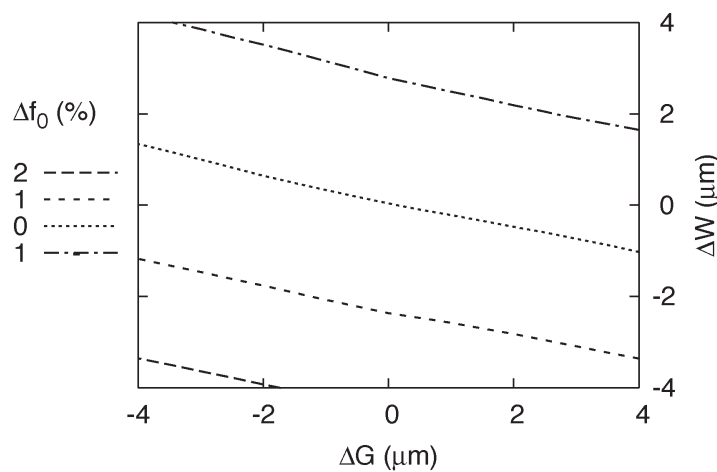

(b)

Fig. 3. Effects of $G-W$ variations on the peak resonant frequency $\Delta f_{0}=\left(f_{\mathrm{r}}-f_{0}\right) / f_{0} * 100$, where $f_{\mathrm{r}}$ is the resulting peak resonant frequency, and $f_{0}$ is the designed resonant frequency. (a) Nominal $G=20 \mu \mathrm{m}$ and (b) nominal $G=50 \mu \mathrm{m}$.

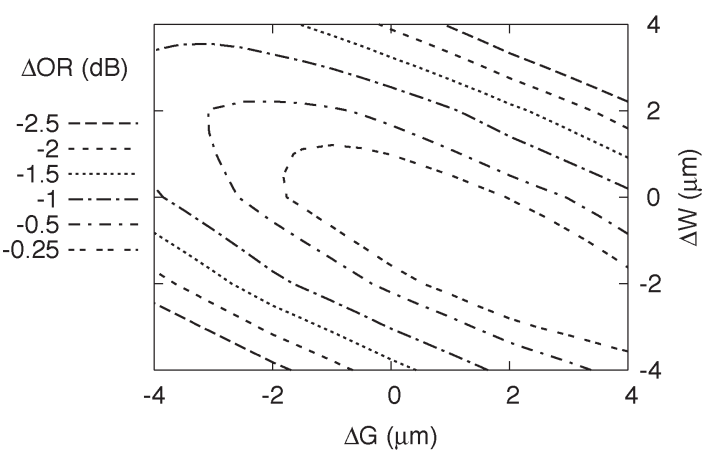

(a)

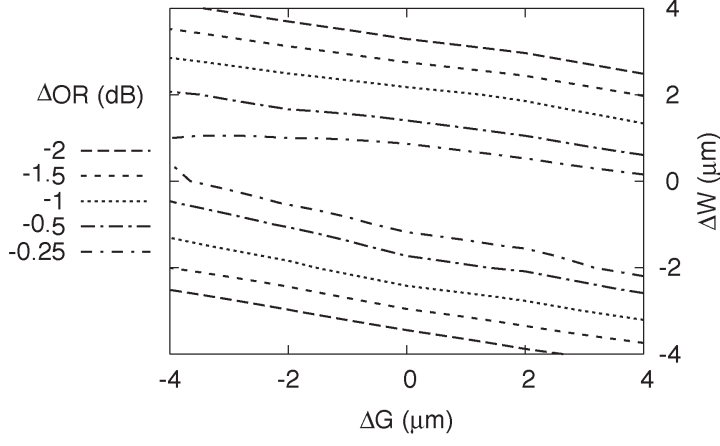

(b)

Fig. 4. Effects of $G-W$ variations on the modulator optical response. (a) Nominal $G=20 \mu \mathrm{m}$ and (b) nominal $G=50 \mu \mathrm{m}$.

electrode loss, and impedance is very small [8]. However, the variation of the hot electrode width has a large effect on the inherent switching voltage. Therefore, at large electrode gap, the modulator optical response is mostly sensitive to the hotelectrode-width variation, as shown in Fig. 4(b), whereas when the gap is small, both electrode gap and width variations have an equal impact on the electrode characteristic impedance, attenuation, and inherent switching voltage. This makes the response sensitive to both gap and width variations.
It can be seen that when the fabricated hot electrode width is smaller than the designed value, the gap should be made larger in order to maintain the peak resonant frequency and the modulator response. Therefore, underetching of the photoresist pattern used for gold plating of the electrodes does not have a detrimental effect on the modulator performance. Whereas overetching of the photoresist can still provide matched resonant frequency, the modulator response is lower than the nominal value. 


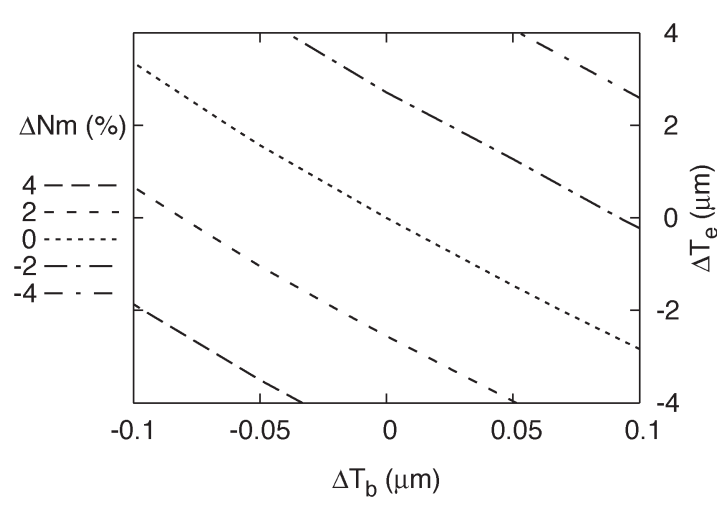

(a)

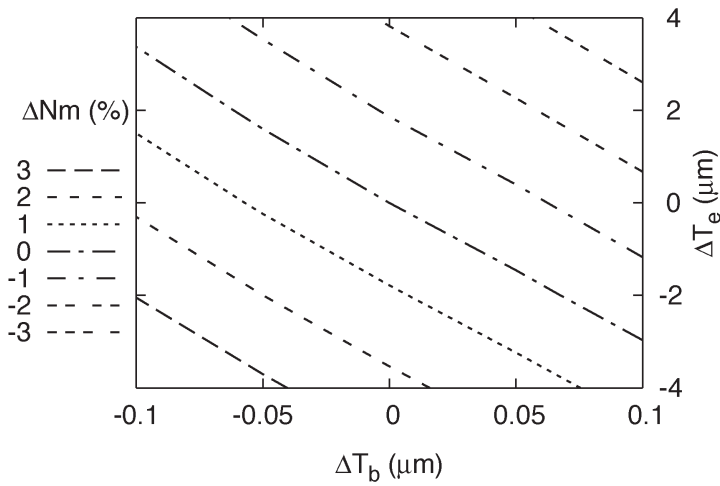

(b)

Fig. 5. Effects of buffer-layer-thickness and electrode-thickness variations on the electrode microwave effective index Nm. (a) Nominal $G=20 \mu \mathrm{m}$ and (b) nominal $G=50 \mu \mathrm{m}$.

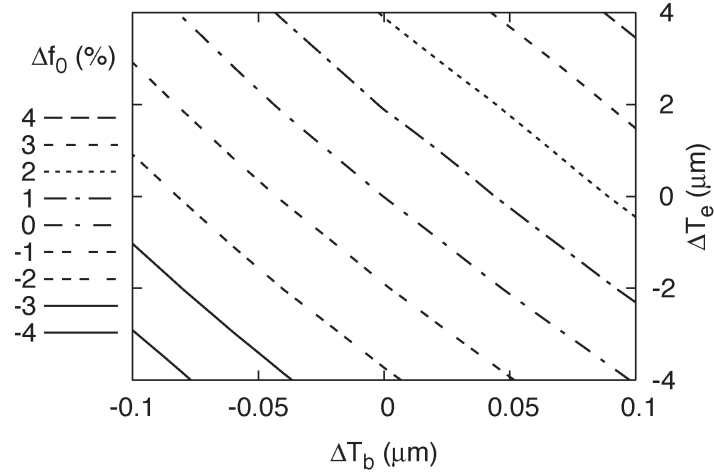

(a)

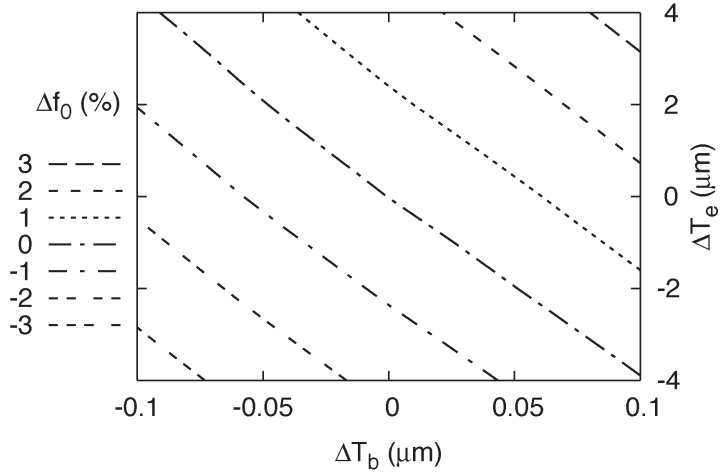

(b)

Fig. 6. Effects of buffer-layer-thickness and electrode-thickness variations on the peak resonant frequency. (a) Nominal $G=20 \mu \mathrm{m}$ and (b) nominal $G=50 \mu \mathrm{m}$.

\section{B. Variations of the Buffer Layer and Electrode Thickness}

The thickness of the buffer layer can be precisely controlled by the in situ monitoring of the layer thickness during the $\mathrm{SiO}_{2}$ deposition process. However, when forming thick electrodes, it is difficult to accurately control the thickness of the electroplated gold layer. In this section, we investigate the effects of the variations of the buffer-layer thickness and electrode thickness on the performance of the resonantly enhanced modulators while keeping the electrode gap and width constant.

Fig. 5 shows how the microwave effective index changes with the variation of the buffer-layer thickness and electrode thickness. The microwave effective index is very sensitive to the change in the thickness of the buffer layer. However, with in situ monitoring, it is possible to achieve the thickness accuracy of $50 \mathrm{~nm}$. As in the case of the gap and width variations of Section III-A, with a wide gap, the effective index is less sensitive to the variation of the buffer layer and electrode thickness.

The behavior of the peak resonant frequency is shown in Fig. 6 when the buffer-layer thickness varies by $\pm 0.1 \mu \mathrm{m}$, and the electrode thickness varies by $\pm 4 \mu \mathrm{m}$. Both wide electrodegap and moderate electrode-gap cases are considered. When the electrode gap is $20 \mu \mathrm{m}$, a $4-\mu \mathrm{m}$ variation of the electrode thickness causes a $2 \%$ change of the peak resonant frequency. If the gap is made wider to $50 \mu \mathrm{m}$, a $1.5 \%$ change of the peak resonant frequency results from the same amount of change of the electrode thickness. The peak resonant frequency of the $50-\mu \mathrm{m}$ nominal gap is less sensitive to the variations of the bufferlayer thickness and gold thickness than the 20- $\mu$ m nominal-gap structure. However, the difference between the two structures is smaller than in the case of electrode gap and width.

In Fig. 7, the sensitivity of the modulator response at the designed frequency to the changes of the buffer-layer thickness and electrode thickness is shown. It is interesting to see that the modulator response in the $50-\mu \mathrm{m}$ gap configuration is more sensitive to the variations of the buffer-layer thickness and electrode thickness than that in the moderate-gap modulator. It was found that the effect of the electrode-thickness variation on the inherent switching voltage is negligible [8]. Therefore, any change to the modulator response only comes from the change of the modulation enhancement due to the resonant cavity. In the small-gap configuration, the electrode-cavity loss is higher than that in the large-gap electrode. Hence, the Q-factor of the resonant cavity of the small-gap modulator is lower than that of the large-gap modulator. Therefore, the shift in the peak resonant frequency causes the modulator response to depart from the designed value more quickly in the case of the largegap modulator. 


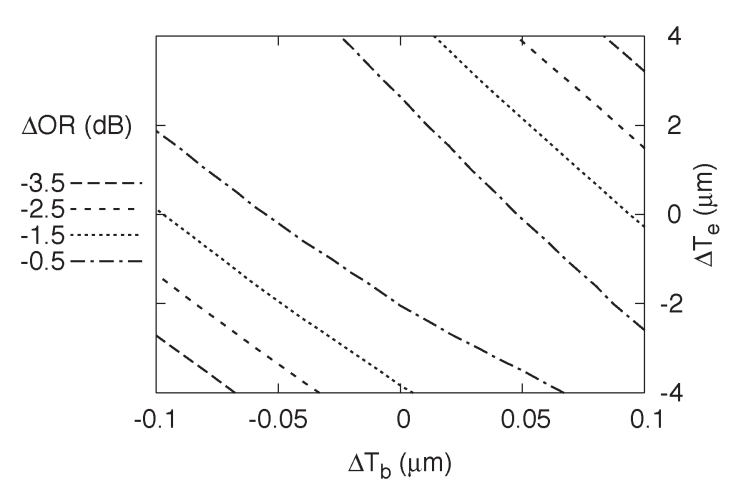

(a)

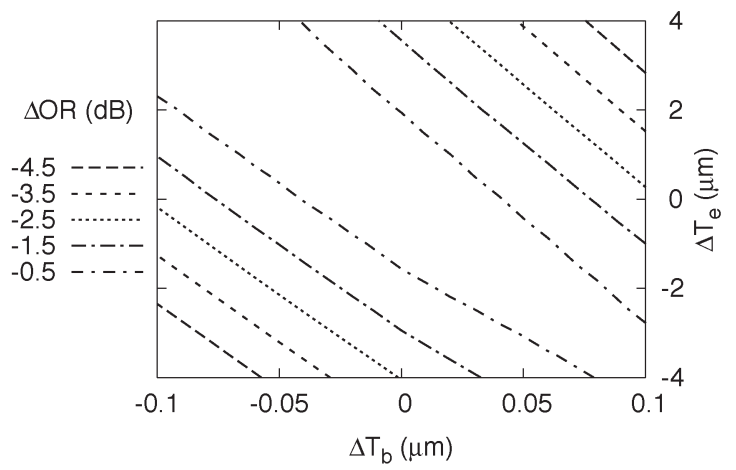

(b)

Fig. 7. Effects of buffer-layer thickness and electrode thickness on the modulator optical response. (a) Nominal $G=20 \mu \mathrm{m}$ and (b) nominal $G=50 \mu \mathrm{m}$.

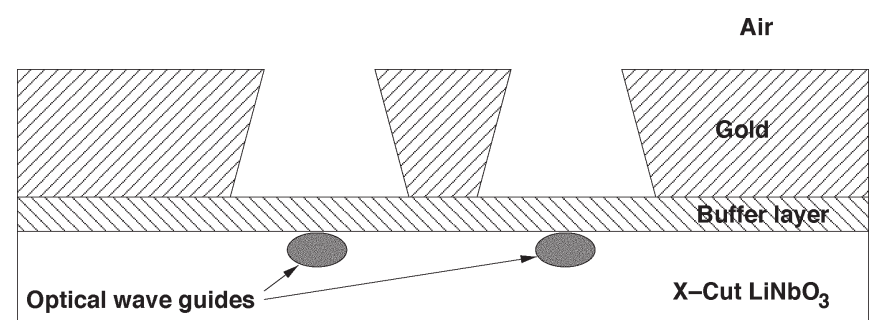

Fig. 8. Trapezoidal CPW electrode.

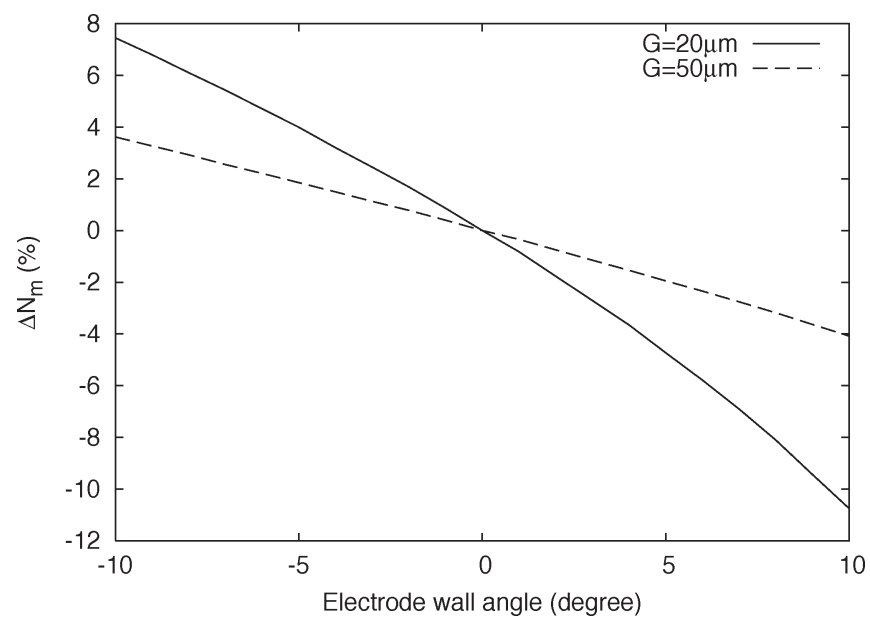

Fig. 9. Variation of microwave effective index as a function of electrode wall angle.

\section{Effect of Electrode Wall Angle}

So far, the electrode walls have been assumed to be perfectly vertical. The optimal electrodes should have a very thick electrode to minimize the electrode loss. With the current electroplating technology, the electrode thickness of $30 \mu \mathrm{m}$ is achievable. However, due to large thickness, the electroplating process will cause the electrode to be in trapezoidal shape, as shown in Fig. 8, with the electrode wall angle up to $10^{\circ}$ [13], [14]. In this section, the effect of the electrode wall angle on the performance of the resonantly enhanced modulator will be considered. As in other investigations, both configurations with small and large gaps will be investigated and compared.

Fig. 9 shows the side-wall-angle dependence of the microwave effective index for both wide-gap and moderate-gap

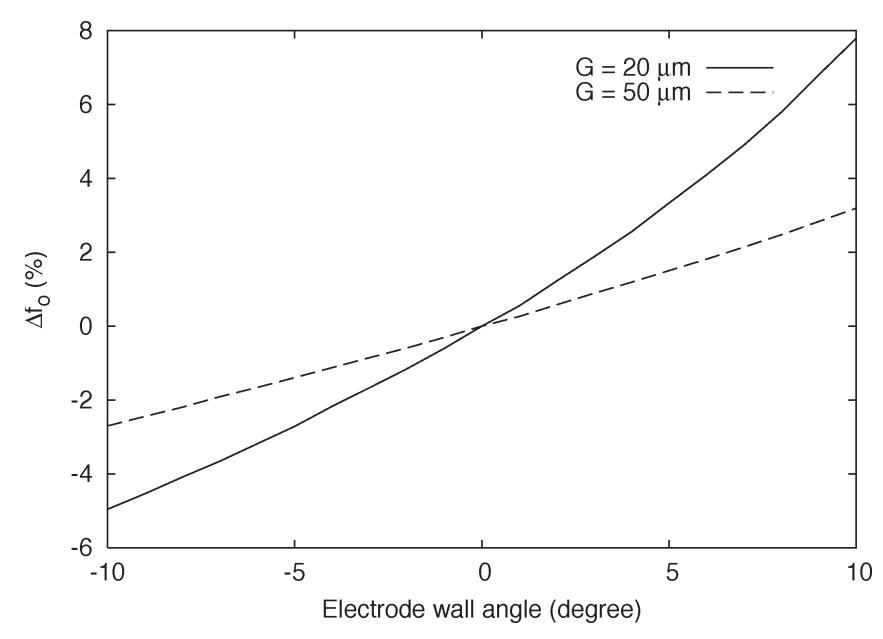

Fig. 10. Error of peak resonant frequency as a function of electrode wall angle.

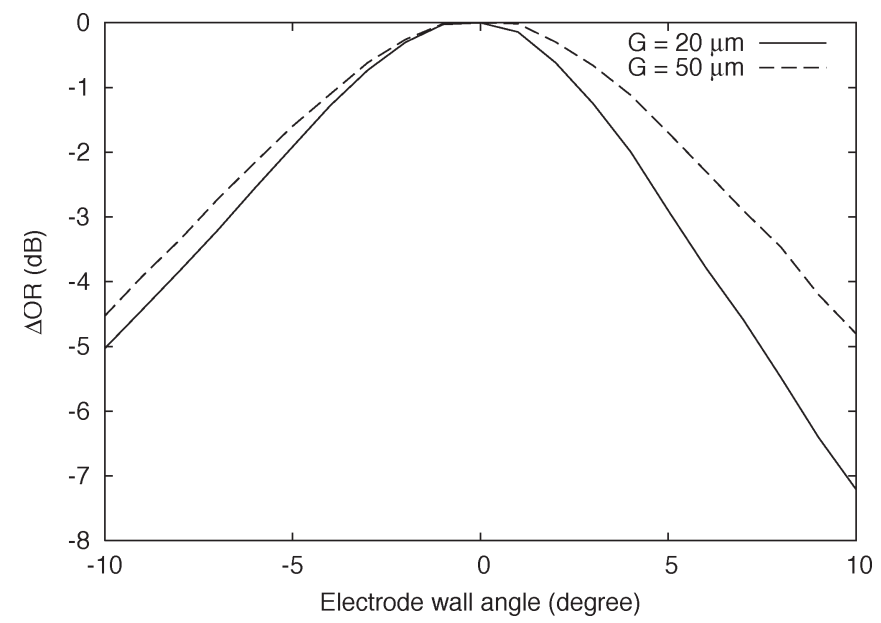

Fig. 11. Variation of modulator response at the designed frequency as a function of electrode wall angle.

electrodes. It can be seen that at a small gap, the microwave effective index varies quickly with the side-wall inclination. The degree of the effective-index variation of the large-gap electrode is much smaller. Fig. 10 shows the sensitivity to the electrode-side-wall variation of the peak resonant frequency, and Fig. 11 shows that of the modulator response at the designed frequency. At large electrode gap of $50 \mu \mathrm{m}$, the 


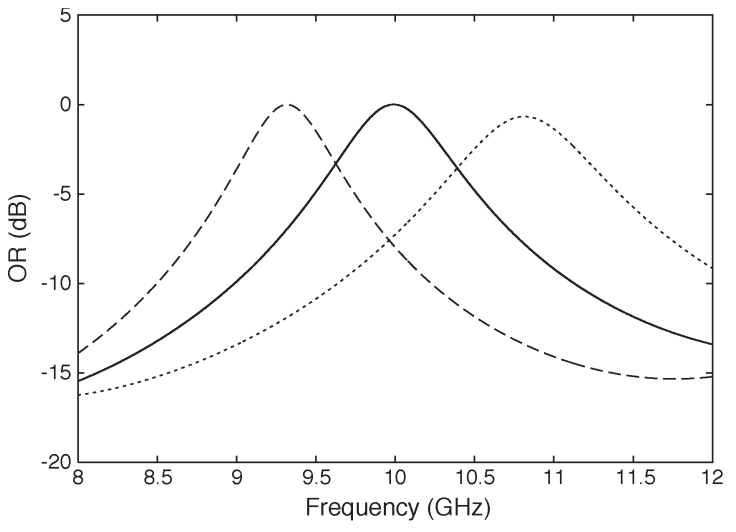

(a)

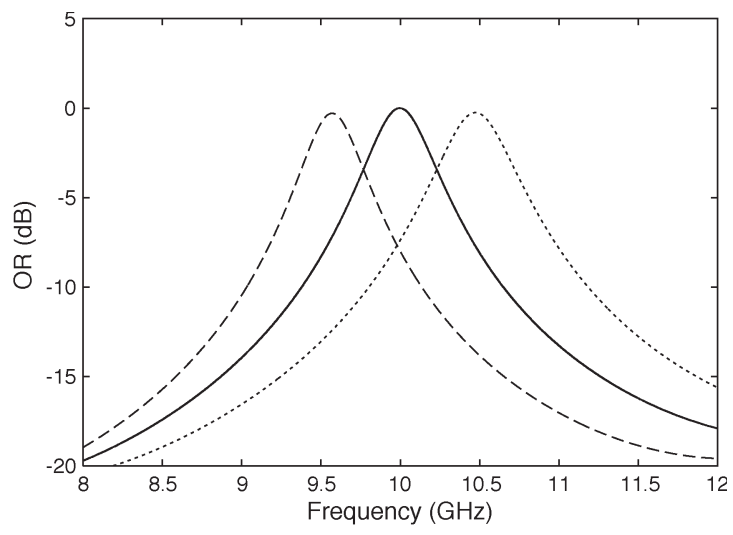

(b)

Fig. 12. Normalized optical response with different variations of electrode dimension and wall angle. (a) Nominal $G=20 \mu \mathrm{m}$ and (b) nominal $G=50 \mu \mathrm{m}$. The solid lines are the responses when all parameters are at their nominal values; the dashed lines are the responses when the gap is increased by $2 \mu \mathrm{m}$, the width is increased by $2 \mu \mathrm{m}$, the gold thickness is reduced by $4 \mu \mathrm{m}$, the buffer-layer thickness is reduced by $0.02 \mu \mathrm{m}$, and the electrode wall angle is $-5^{\circ}$; and the dotted lines are the responses when the gap is reduced by $2 \mu \mathrm{m}$, the width is reduced by $2 \mu \mathrm{m}$, the gold thickness is increased by $4 \mu \mathrm{m}$, the buffer-layer thickness is increased by $0.02 \mu \mathrm{m}$, and the electrode wall angle is $5^{\circ}$.

electrode can be electroplated in a single layer without any degradation to the performance. For a small electrode gap, multilayer electroplating, which is a more expensive process, may be required to keep the modulator performance within the acceptable tolerance.

\section{Random Variations of Parameters}

In previous sections, the effect of the variation of individual electrode dimension on the performance of the resonantly enhanced modulator has been analyzed. This section analyzes how the performance is affected if the variations in all dimensions are taken into account.

First, the worst-case scenario, in which all dimensions are varied such that the error in peak resonant frequency due to the errors in each dimension have the same sign and thus add cumulatively, producing the most extreme results, is considered. Fig. 12(a) and (b) presents the frequency-dependent optical response of these cases together with that of the nominal cases shown in for nominal gaps of 20 and $50 \mu \mathrm{m}$, respectively. The optical responses were normalized to the responses at $10 \mathrm{GHz}$. The results of Fig. 12(a) and (b) confirm the conclusions of the previous sections that the peak resonant frequency of the modulator with 20- $\mu \mathrm{m}$ gap departs from the designed frequency more quickly than of the 50- $\mu \mathrm{m}$ nominal-gap modulator with the same variation of electrode dimensions. It was observed in Section III-B that the modulator optical response at the designed operating frequency of the 50- $\mu \mathrm{m}$ nominal-gap modulator is more sensitive to the variation of the buffer-layer thickness and electrode thickness than that of the modulator with the $20-\mu \mathrm{m}$ gap. However, if the variations of all electrode dimensions, are considered, the errors in the optical responses of both modulators are almost the same.

Next, a Monte Carlo analysis was performed to assess the sensitivity of the resonantly enhanced modulator with respect to random variations of electrode physical dimensions. Normal distributions were assumed for the variations of all

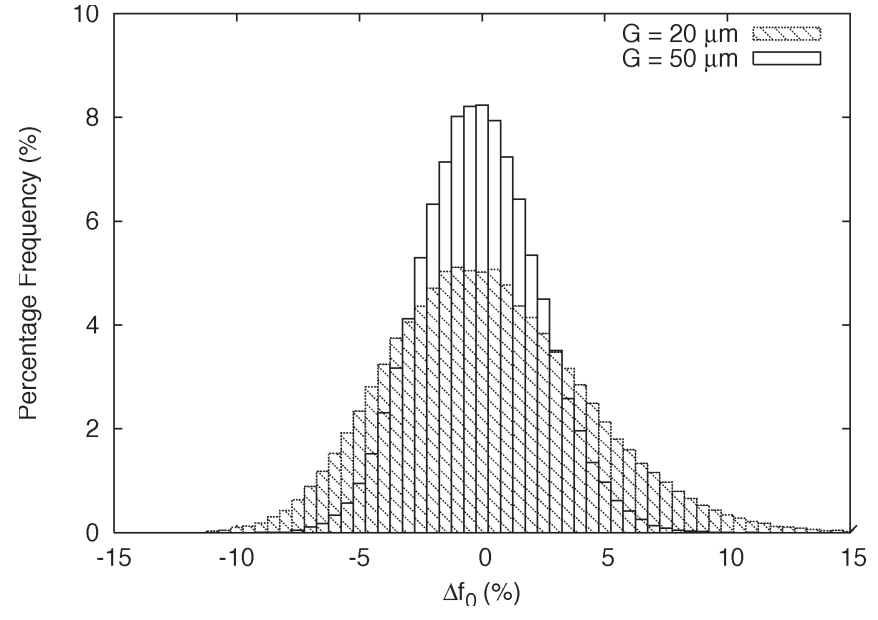

Fig. 13. Percentage-frequency histogram of the errors of peak resonant frequency with the random variations of electrode dimensions and wall angle.

electrode dimensions, including the electrode wall angle. The standard deviation of each dimension was electrode gap: $2 \mu \mathrm{m}$, width: $2 \mu \mathrm{m}$, gold thickness: $4 \mu \mathrm{m}$, buffer-layer thickness: $0.02 \mu \mathrm{m}$, and wall angle: $5^{\circ}$. The number of devices simulated was 32000 for each case of nominal electrode gap.

The relative-frequency histograms of the errors of the peak resonant frequency for the 20- and 50- $\mu \mathrm{m}$ nominal-gap modulators are shown in Fig. 13. There are few noticeable features that can be identified from Fig. 13. As discussed previously, the variation of the peak resonant frequency of the largegap modulator is much smaller than that of the small-gap modulator. The distributions of the peak resonant-frequency variations follow approximately normal distributions with the standard deviations of $4 \%$ and $2.4 \%$ for the $20-$ and $50-\mu \mathrm{m}$ nominal-gap modulators, respectively. Out of 32000 simulated devices, $60 \%$ of the devices with a $50-\mu \mathrm{m}$ nominal gap have the peak resonant frequency within $\pm 2 \%$ of the designed value, whereas the number for the $20-\mu \mathrm{m}$ nominal-gap modulators is only $39 \%$. 


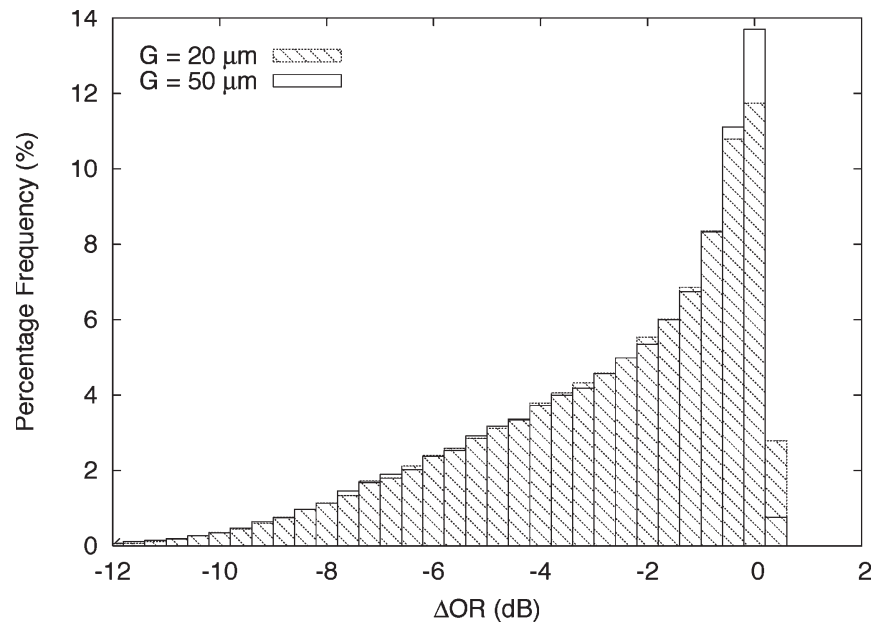

Fig. 14. Percentage-frequency histogram of the variations of optical response at the designed frequency with the random variations of electrode dimensions and wall angle.

Fig. 14 shows the relative-frequency histograms of the variations of the optical response at the designed frequency for both 20 - and 50- $\mu$ m nominal-gap modulators. Approximately $61.5 \%$ of the 32000 simulated devices have the optical response at the designed operating frequency degrading by $3 \mathrm{~dB}$ or less from the designed value for both nominal-gap structures.

\section{DISCUSSIONS}

In the previous sections, the effects of the variations of electrode cross-sectional dimensions and electrode wall angle on the performance of the modulators with resonant electrode structures have been analyzed. The analysis reveals that the modulator performance is less sensitive to the variations due to the fabrication process if the electrode gap is made large. With a large electrode gap, the modulators can be fabricated quickly without much penalty to the performance. It is possible, with such large structures, that low-cost fabrication techniques, such as laminated photoresists, could be used.

In these investigations, only the variations of the electrode cross-sectional dimensions have been considered. The variations of the optical-waveguide-fabrication conditions have not been investigated in this paper. The fluctuation of the optical waveguide only affects the inherent switching voltage, which leads to the overall modulation efficiency. It has no effect on the resonant behavior of the modulator; hence, a variation in optical waveguides will impact a yield in a similar manner to the broadband modulator.

\section{CONCLUSION}

We have presented an analysis of the sensitivity to the fabrication tolerances of resonantly enhanced modulators on $\mathrm{X}$-cut $\mathrm{LiNbO}_{3}$ with $\mathrm{CPW}$-electrode structure. FEM simulation has been employed to characterize the electrodes with different variations in dimensions. The effects of the electrode wall angle were also considered. It has been shown that a largegap electrode is a preferable choice for resonantly enhanced modulators because modulators with a large electrode gap not only provide a better resonant enhancement but also are less sensitive to the fluctuations of the electrode cross-sectional dimensions and shape.

\section{REFERENCES}

[1] A. Nirmalathas, D. Novak, C. Lim, R. Waterhouse, and D. Castleford, "Fiber networks for wireless applications," in Proc. LEOS Annu. Meeting Conf., 2000, vol. 1, pp. 35-36.

[2] X. Yao and L. Maleki, "Optoelectronic oscillator for photonic systems," IEEE J. Quantum Electron., vol. 32, no. 7, pp. 1141-1149, Jul. 1996.

[3] A. Lindsay, G. Knight, and S. Winnall, "Photonic mixers for wide bandwidth RF receiver applications," IEEE Trans. Microw. Theory Tech., vol. 43, no. 9, pp. 2311-2317, Sep. 1995.

[4] M. Dennis, W. Burns, T. Carruthers, and I. N. Duling, "Eight-to-one demultiplexing of 100-Gb/s TDM data using $\mathrm{LiNbO}_{3}$ Sagnac interferometer modulators," in Proc. OFC Conf. Exhibit Tech. Dig., 1998, pp. 110-112.

[5] T. Kawanishi, S. Oikawa, K. Higuma, Y. Matsuo, and M. Izutsu, "Resonant electrode structures for band-operation-type optical modulators," in Proc. SPIE, 2002, vol. 4872, pp. 1-10.

[6] R. Krähenbühl and M. Howerton, "Investigations on short-path-length high-speed optical modulators in $\mathrm{LiNbO}_{3}$ with resonant-type electrodes," J. Lightw. Technol., vol. 19, no. 9, pp. 1287-1297, Sep. 2001.

[7] S. Oikawa, T. Kawanishi, K. Higuma, Y. Matsuo, and M. Izutsu, "Doublestub structure for resonant-type optical modulators using $20-\mu$ m-thick electrode," IEEE Photon. Technol. Lett., vol. 15, no. 2, pp. 221-223, Feb. 2003.

[8] T. G. Nguyen, A. Mitchell, and Y. S. Visagathilagar, "Investigation of resonantly enhanced modulators on $\mathrm{LiNbO}_{3}$ using FEM and numerical optimization technique," J. Lightw. Technol., vol. 22, no. 2, pp. 526-533, Feb. 2004.

[9] Y. Visagathilagar, A. Mitchell, and R. Waterhouse, "Fabry-Pérot type resonantly enhanced Mach-Zehnder modulator," in Proc. Int. Top. Meet. MWP, 1999, vol. 1, pp. 17-20.

[10] Y. S. Visagathilagar, T. G. Nguyen, A. Mitchell, and M. W. Austin, "Systematic design approach for optimised resonantly enhanced MachZehnder modulators," J. Lightw. Technol., vol. 24, no. 1, pp. 555-562, Jan. 2006.

[11] J. Jin, The Finite Element Method in Electromagnetics. Hoboken, NJ: Wiley, 1993.

[12] A. Mitchell, "Efficient PML boundaries for anisotropic waguide simulations using the finite element method," Ph.D. dissertation, School Elect. Comput. Eng., RMIT Univ., Melbourne, Australia, 1999.

[13] G. Ghione, M. Goano, G. Madonna, G. Omegna, M. Pirola, S. Bosso, D. Frassati, and A. Perasso, "Microwave modeling and characterization of thick coplanar waveguides on oxide-coated lithium niobate substrates for electrooptical applications," IEEE Trans. Microw. Theory Tech., vol. 47, no. 12, pp. 2287-2293, Dec. 1999.

[14] H. W. Chong, "High performance lithium niobate optical modulators using thick electrodes and surface machining," Ph.D. dissertation, School Elect. Comput. Eng., RMIT Univ., Melbourne, Australia, 2002.

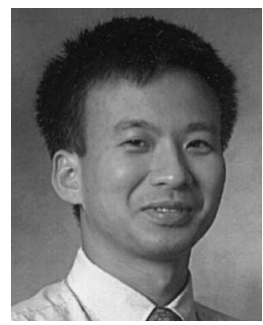

Thach Giang Nguyen was born in Quang Tri, Vietnam, in 1975. He received the B.Eng. degree in communication engineering (with first-class honors) from the Royal Melbourne Institute of Technology (RMIT), Melbourne, Australia, in 1998. He is currently working toward the Ph.D. degree in communication engineering at the School of Electrical and Computer Engineering, RMIT.

From 1999 to 2002, he was a Design Engineer with Vietnam Telecommunications Technology Co. (VITECO), Vietnam Post and Telecommunications Group (VNPT), Hanoi, Vietnam. His research interests are investigation of specialized optical modulators and modeling of integrated photonic devices. 


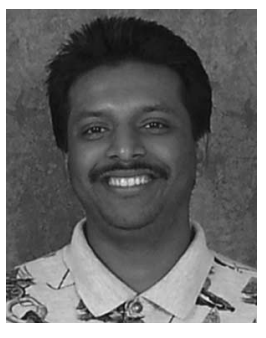

Yuvaraja S. Visagathilagar (S'93-A'02-M'02) was born in Colombo, Sri Lanka, on July 23, 1972. $\mathrm{He}$ received the B.Eng. degree in communications (with first-class honors) and the Ph.D. degree in communication engineering from the Royal Melbourne Institute of Technology (RMIT), Melbourne, Australia, in 1996 and 2003, respectively.

From 1999 to 2002, he was a Researcher undertaking the development of high-speed optical modulators as part of a defense contract. During 2002-2004, he was undertaking research at the Australian Photonics Cooperative Research Centre (CRC), RMIT University. $\mathrm{He}$ is currently a Research Fellow with the School of Electrical and Computer Engineering, RMIT University, where he investigates broadband and specialized optical modulators, in particular, narrowband and resonantly enhanced modulators. His research interests are in theoretical and experimental investigation of microwave and microwave/photonic devices as well as integrating these devices with microwave devices/antennas for telecommunication and defense.

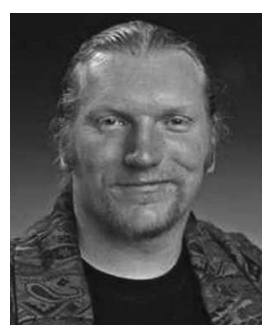

Arnan Mitchell (S'97-M'00) was born in Dublin, Ireland, on February 20, 1973. He received the B.Tech. degree in optoelectronics (with honors) from Macquarie University, Sydney, Australia, in 1993 and the $\mathrm{Ph} . \mathrm{D}$. degree in communication engineering from the Royal Melbourne Institute of Technology (RMIT), Melbourne, Australia, in 1999.

$\mathrm{He}$ was an Australian Photonics CRC Research Fellow at RMIT, investigating broadband and specialized integrated optical modulators and radio-frequency (RF) photonic components for communications and signal-processing applications. Currently, he is a Senior Lecturer in the School of Electrical and Computer Engineering, RMIT. He maintains an active interest in the research of numerical methods required for the design of RF photonic integrated devices. 